\title{
Macrophage depletion: a potential immunomodulator treatment of endometriosis-associated pain?
}

\author{
Thomas M. D’Hooghe ${ }^{1,2,3}$, Arne Vanhie ${ }^{1,4}$, Valerie A. Flores ${ }^{2}$, Hugh S. Taylor ${ }^{2}$ \\ ${ }^{1}$ Research Group Reproductive Medicine, Department of Development and Regeneration, Organ Systems, Group Biomedical Sciences, KU Leuven \\ (University of Leuven), Belgium; ${ }^{2}$ Department of Obstetrics, Gynecology and Reproductive Sciences Yale School of Medicine, New Haven, CT, \\ USA; ${ }^{3}$ Vice-President and Head, Global Medical Affairs Fertility, Research and Development, Merck Healthcare KGaA, Darmstadt, Germany; \\ ${ }^{4}$ Leuven University Fertility Center and Department of Obstetrics and Gynecology, University Hospitals Leuven, Leuven, Belgium \\ Correspondence to: Thomas M. D’Hooghe, MD, PhD. Research Group Reproductive Medicine, Department of Development and Regeneration, \\ Organ Systems, Group Biomedical Sciences, KU Leuven (University of Leuven), p/a Secretarial Office Department Obstetrics and Gynecology, \\ University Hospitals Leuven, UZ Gasthuisberg, Herestraat 49, 3000 Leuven, Belgium. Email: thomas.dhooghe@kuleuven.be. \\ Comment on: Forster R, Sarginson A, Velichkova A, et al. Macrophage-derived insulin-like growth factor-1 is a key neurotrophic and nerve-sensitizing \\ factor in pain associated with endometriosis. FASEB J 2019;33:11210-22.
}

Submitted Apr 24, 2020. Accepted for publication May 14, 2020.

doi: 10.21037/atm-2020-98

View this article at: http://dx.doi.org/10.21037/atm-2020-98

In a recent paper published in FASEB F, Forster et al. provide compelling evidence to support the role of macrophages in mediating endometriosis-associated pain (1). Deposition of ectopic endometrium elicits an immune response-there are increased local accumulation of white blood cells, with macrophages dominating as the resident immune cell population $(2,3)$. As such, inflammation is a prominent feature of endometriosis, with production of pro-inflammatory cytokines and abnormal immune cell distribution (4). In this article, Forster et al. (1) note that ectopic endometrium recruits sensory nerve fibers that innervate lesions and can be activated by the proinflammatory environment. Previous reports have found neurologic manifestations to coincide with endometriosis. Women with endometriosis are often hypersensitive to pain, which is postulated to be the result of ongoing inflammation, leading to sensitization of the nociceptive system. This sensitization decreases pain thresholds and amplifies sensory input, resulting in central pain sensitization (CPS) (5-7). In a murine model of endometriosis, $\mathrm{Li}$ et al. found that mice with endometriosis demonstrated increased sensitivity to painful stimuli and altered neurophysiologic changes in the amygdala, a region of the brain involved in pain, anxiety, and depression (8). Furthermore, the same group demonstrated altered gene expression in the insula, amygdala, and hippocampus (additional regions of the brain associated with pain, anxiety and depression) (8). Forster et al. (1) provide additional insight regarding potential mechanisms of altered sensitization, suggesting in addition to the brain mediated sensitization reported by $\mathrm{Li}$ et al. (8), there is also a peripheral nerve sensitization. The authors note that inflammatory cytokines interact with peripheral sensory afferents such that there is nerve sensitization-resulting in increased excitability and triggering hypersensitivity. In recognizing that macrophages play active roles in pain through production of pronociceptive molecules that can activate nerves, and noting that macrophages are key players in endometriosis, they hypothesized that macrophages contribute to endometriosis-associated pain by secreting factors promoting nerve growth and sensitization. Utilizing a validated murine model of menstruation to induce endometriosis in immunocompetent mice (9), peritoneal fluid, and ectopic endometrium samples, they sought to determine how macrophages contribute to endometriosisassociated pain.

Forster et al. (1) demonstrated that depleting macrophages in a murine model of endometriosis resulted in decreased endometriosis-associated pain-as evidenced by attenuation of spontaneous grooming and mechanical hyperalgesia. They found decreased expression of Cox-2 in the brain, and decreased Cox- 2 and TNF- $\alpha$ in the spinal cords of these mice; equally important was a reduction in 
lesion number. Macrophage-derived insulin-like growth factor 1 (IGF-1), a signaling factor for nerve outgrowth and sensitization, was also decreased. As macrophages have diverse roles in both the normal immune response and disease states, the function of macrophages in women with endometriosis was assessed. In the peritoneal fluid of women with endometriosis, IGF-1 levels were elevated when compared to women without endometriosis, and this was positively correlated with pain scores. In collected human endometriotic lesions they found macrophages expressing IGF-1. Furthermore, in macrophages activated with peritoneal fluid from women with endometriosis (endometriosis-associated macrophages), there was increased expression of IGF-1. To better characterize the role of IGF-1 in endometriosis, the IGF-1 receptor was inhibited in their murine model, and this resulted in decreased mechanical hyperalgesia. They also found that addition of an inhibitor of the IGF-1 receptor reversed both neurogenesis (mediated by endometriosis associated macrophages and/or addition of recombinant IGF-1) in rat dorsal root ganglion and sensitization in sensory neurons. In summary, this study provides convincing evidence that macrophages are involved in pain symptoms of women with endometriosis-in part mediated by IGF-1. Inhibition of the IGF-1 receptor can be considered as a potential novel therapeutic target.

Their study is well-designed, and the results are intriguing; utilizing a combination of human and animal models allowed them to test theories and potential therapeutic targets. In addition, they build upon their previous work with respect to noting increased expression of nociceptive and inflammatory markers in the brains and spinal cord of mice, and were able to identify the involvement of macrophages in these processes $(10,11)$. While they demonstrate compelling data for the role of macrophages in mediating pain in endometriosis, it is important to recognize some limitations.

With respect to the animal data, we would like to point out the following comments/queries.

Firstly, the design of the study does not allow to determine whether liposomal clodronate/macrophage depletion actually resulted in endometriosis lesion regression. This limitation is related to the variable success rate in induction of endometriosis after intraperitoneal injection of menstrual-like endometrium from donor mice, and to the fact that induction success can only be assessed by post-mortem examination. In the original description of the mouse model (9) the success rate of endometriosis induction was $83 \%$. In the paper by Forster et al. (1) the success rate of endometriosis induction is $71 \%$ (22 out of 31 induced animals), which is comparable to the $83 \%$ described above (Fisher's exact test, $\mathrm{P}=0.49$ ). Twentyone days after induction of endometriosis, 31 animals were randomly allocated to the nondepleted saline group $(n=14)$ or to the deleted liposomal clodronate group $(n=17)$ without explanation for the unbalanced randomization (a distribution of $15 v s .16$ animals would have been expected). In the nondepleted saline group the prevalence of confirmed endometriosis (presence of at least one lesion on day 28 of the experiment) was $93 \%$ (13/14), significantly higher than the $53 \%(9 / 17)$ observed in the depleted liposomal clodronate group (Fisher's exact test, $\mathrm{P}=0.02$ ). The animals where no lesions were detected at the end of the experiment either never had endometriosis because of unsuccessful induction of endometriosis or the lesions had disappeared due to the liposomal clodronate/macrophage depletion. Obviously, the other results (analysis of peritoneal fluid, brain biopsies, behavioural tests, etc.) can equally be explained by either an effect of macrophage depletion in the liposomal clodronate group or by the failure of induction of endometriosis in this group with the absence of endometriosis lesions in 47\% (10/17). Therefore, it would be interesting and scientifically more accurate to perform a separate analysis only including the animals that had histologically proven endometriosis on day 28. Similarly, IGF-1 R-inhibitor appeared to reduce pain, but it is not clear if/how this was related to an effect on endometriosis lesions, as no data are presented how many of the 24 mice (12 treated with inhibitor, 12 with placebo vehicle) actually were confirmed to have endometriosis.

Secondly, there are several important gaps in the way data are presented and analyzed. It is not clear how many endometriosis lesions were present, where they were located and how they were selected for biopsy and for subsequent analysis. It is also not clear why brain/spinal cord assessment was done in only $7 / 17$ depleted and in $7 / 14$ nondepleted animals. Were the differences shown in figure $2 \mathrm{G}$ and $2 \mathrm{H}$ observed between depleted and non-depleted endometriosis lesions (according to Figure legend) or between mice with endometriosis versus controls (according to statement in text)? Which method was used to analyze the behavior tests (done 4 times, $1 \times$ baseline, 3 times after depletion or nondepletion) resulting in figure 2 , sections I to $\mathrm{L}$ ? It is not clear if IGF mRNA could be detected in endometriosis lesions: in figure $4 \mathrm{~F}$ and $4 \mathrm{G}$, IGF positive macrophages were shown to be present in mice endometriosis lesions 
(figure 4F), but IGF mRNA data (figure 4G) were shown only for peritoneum biopsies, not for endometriosis lesions. Finally, the statement that detectable molecular markers of inflammatory pain in the nervous system of mice with endometriosis can be attenuated by macrophage depletion is correct for COX2 in both spinal cord and brain, but not for TNFalpha levels that were not significantly decreased.

In addition, the human subject data are limited by the low sample size $(\mathrm{n}=21$, including 13 women with pain and endometriosis and I women with pain without endometriosis), the absence of a control group without pelvic pain, and the lack of more detailed information on endometriosis phenotypes (ASRM endometriosis stage and score; types and locations of lesions) and sampling selection (were endometriosis lesions and peritoneal fluid obtained from the same women with endometriosis?). For example, while the authors found that IGF-1 levels are elevated in endometriosis subjects compared with controls, and that IGF-1 levels were positively correlated with pain scores, the correlation is weak. Endometriosis is a heterogeneous disease and important clinical parameters are not addressed. Although the data are compelling, and in line with prior work assessing disease severity and markers of macrophage activity (12), their value is limited by the small and homogeneous subject pool. It is equally important to recognize that administration of a macrophage depleting agent or administration of an agent involved in blocking IGF-1 binding to its receptor cannot be globally administered due to the multiple functions of macrophages and IGF-1 respectively. The authors acknowledge this limitation and suggest an alternative approach targeting "disease-promoting" macrophages as a future therapeutic option for women with endometriosis.

Altered miRNA expression also contributes to the inflammatory environment of endometriosis (13), providing another mechanism by which endometriosis induces a persistent inflammatory state, as well as altered pain sensitization. We and others have demonstrated altered levels of miRs in women with endometriosis (14-19). In a study by Nematian et al., pro-inflammatory cytokine levels (TNF- $\alpha$, IL-1 $\beta$, and IL- 6 ) in the peripheral blood of patients with and without endometriosis showed correlation with serum levels of miR-125-5p and let-7b (13). Furthermore, transfection of macrophages with miR125 mimic and miR-Let7b inhibitor resulted in increased TNF- $\alpha$, IL-1 $\beta$, IL- 6 , and IL- 8 levels while transfection with miR-Let7b mimic resulted in significantly decreased levels of TNF- $\alpha$ and IL-8 (13). Thus, aberrant levels of miR-
$125-5 \mathrm{p}$ and miR-Let7b promote the inflammatory milieu of endometriosis, likely through altering macrophagemediated release of pro-inflammatory cytokines. Peritoneal macrophages are known to be increased in endometriosis, stimulate endometriotic lesion growth and mediate the pain response commonly seen in endometriosis. Forster et al. (1) and $\mathrm{Li}$ et al. (8) both demonstrate altered pain sensitivity in endometriosis. Through Forster et al.'s work (1), a role for macrophages not only in promoting the inflammatory environment of endometriosis, but also in promoting nerve growth and pain sensitization in endometriosis, is clear.

The inflammatory nature of endometriosis contributes to the systemic effects of the disease, and highlight the need for novel, nonhormonal medical therapy. As Forster et al. discuss (1), one consideration is to target "disease promoting" macrophages with a potential reduction in rodent pain behavior, extending earlier studies in the rat model by Haber and colleagues that macrophage depletion by intraperitoneal (IP) injection of liposomal alendronate (LA) can inhibit endometriosis lesion initiation and growth (20). Other considerations could be to inactivate or deplete B cells, as polyclonal activation of $\mathrm{B}$ cells and the presence of antiendometrial autoantibodies have been described in a large proportion of women with endometriosis. It has recently been shown that skewing activated B cells toward regulatory B cells (Bregs) by Bruton's tyrosine kinase (Btk) inhibition using Ibrutinib prevents endometriosis progression in mice while B cell depletion using an anti-CD20 antibody has no effect (21). Overall, these novel treatment approaches utilize immune modulators for treating this chronic, debilitating disease (22). As macrophages also have a normal function in wound healing and the immune response (23), future work will need to focus on specifically targeting aberrant macrophage function, so as to not compromise the normal immune response, and to confirm effectiveness, as well as overall and reproductive safety of immunomodulation in preclinical animal models close to humans such as the baboon model $(22,24,25)$.

\section{Acknowledgments}

Funding: None.

\section{Footnote}

Provenance and Peer Review: This article was commissioned and reviewed by the Section Editor Hengwei Liu, MD, $\mathrm{PhD}$ (Department of Obstetrics and Gynecology, Zhongnan 
Hospital of Wuhan University, Wuhan, China).

Conflicts of Interest: All authors have completed the ICMJE uniform disclosure form (available at http://dx.doi. org/10.21037/atm-2020-98). VAF reports personal fees from Abbvie, personal fees from Ferring, outside the submitted work. TMD reports other from null, during the conduct of the study; other from null, outside the submitted work. TMD is Vice President and Head Global Medical Affairs Fertility, Research and Development, Merck Healthcare KGaA, Darmstadt, Germany. He is also a Professor in Reproductive Medicine and Biology at the Department of Development and Regeneration, Group Biomedical Sciences, KU Leuven (University of Leuven), Belgium, and an Adjunct Professor at the Department of Obstetrics and Gynecology in the University of Yale, New Haven, USA. HST reports grants and personal fees from Abbvie, personal fees from Bayer, personal fees from Obseva, personal fees from Dot Lab, personal fees from Forendo, outside the submitted work. The author has no other conflicts of interest to declare.

Ethical Statement: All authors are accountable for the full content of this paper, and confirm that they have written this paper with full integrity, interpreting the available data critically and as accurately as possible.

Open Access Statement: This is an Open Access article distributed in accordance with the Creative Commons Attribution-NonCommercial-NoDerivs 4.0 International License (CC BY-NC-ND 4.0), which permits the noncommercial replication and distribution of the article with the strict proviso that no changes or edits are made and the original work is properly cited (including links to both the formal publication through the relevant DOI and the license). See: https://creativecommons.org/licenses/by-nc-nd/4.0/.

\section{References}

1. Forster R, Sarginson A, Velichkova A, et al. Macrophagederived insulin-like growth factor-1 is a key neurotrophic and nerve-sensitizing factor in pain associated with endometriosis. FASEB J 2019;33:11210-22.

2. Grandi G, Mueller MD, Bersinger NA, et al. The association between progestins, nuclear receptors expression and inflammation in endometrial stromal cells from women with endometriosis. Gynecol Endocrinol 2017;33:712-5.
3. Bruner-Tran KL, Herington JL, Duleba AJ, et al. Medical management of endometriosis: emerging evidence linking inflammation to disease pathophysiology. Minerva Ginecol 2013;65:199-213.

4. Lebovic DI, Mueller MD, Taylor RN. Immunobiology of endometriosis. Fertil Steril 2001;75:1-10.

5. Bajaj P, Madsen H, Arendt-Nielsen L. Endometriosis is associated with central sensitization: a psychophysical controlled study. J Pain 2003;4:372-80.

6. Chen LC, Hsu JW, Huang KL, et al. Risk of developing major depression and anxiety disorders among women with endometriosis: A longitudinal follow-up study. J Affect Disord 2016;190:282-5.

7. Lagana AS, Condemi I, Retto G, et al. Analysis of psychopathological comorbidity behind the common symptoms and signs of endometriosis. Eur J Obstet Gynecol Reprod Biol 2015;194:30-3.

8. Li T, Mamillapalli R, Ding S, et al. Endometriosis alters brain electro-physiology, gene expression and increased pain sensitization, anxiety, and depression in female mice. Biol Reprod 2018;99:349-59.

9. Greaves E, Cousins FL, Murray A, et al. A novel mouse model of endometriosis mimics human phenotype and reveals insights into the inflammatory contribution of shed endometrium. Am J Pathol 2014;184:1930-9.

10. Greaves E, Temp J, Esnal-Zufiurre A, et al. Estradiol is a critical mediator of macrophage-nerve cross talk in peritoneal endometriosis. Am J Pathol 2015;185:2286-97.

11. Greaves E, Collins F, Esnal-Zufiaurre A, et al.

Estrogen receptor (ER) agonists differentially regulate neuroangiogenesis in peritoneal endometriosis via the repellent factor SLIT3. Endocrinology 2014;155:4015-26.

12. Arici A, Oral E, Attar E, et al. Monocyte chemotactic protein-1 concentration in peritoneal fluid of women with endometriosis and its modulation of expression in mesothelial cells. Fertil Steril 1997;67:1065-72.

13. Nematian SE, Mamillapalli R, Kadakia TS, et al. Systemic Inflammation Induced by microRNAs: EndometriosisDerived Alterations in Circulating microRNA 125b-5p and Let-7b-5p Regulate Macrophage Cytokine Production. J Clin Endocrinol Metab 2018;103:64-74.

14. Cosar E, Mamillapalli R, Ersoy GS, et al. Serum microRNAs as diagnostic markers of endometriosis: a comprehensive array-based analysis. Fertil Steril 2016;106:402-9.

15. Joshi NR, Miyadahira EH, Afshar Y, et al. Progesterone Resistance in Endometriosis Is Modulated by the Altered Expression of MicroRNA-29c and FKBP4. J Clin 
Endocrinol Metab 2017;102:141-9.

16. Joshi NR, Su RW, Chandramouli GV, et al. Altered expression of microRNA-451 in eutopic endometrium of baboons (Papio anubis) with endometriosis. Hum Reprod 2015;30:2881-91.

17. Nothnick WB, Falcone T, Joshi N, et al. Serum miR451a Levels Are Significantly Elevated in Women With Endometriosis and Recapitulated in Baboons ( Papio anubis) With Experimentally-Induced Disease. Reprod Sci 2017;24:1195-202.

18. Li R, Qiao J, Wang L, et al. MicroRNA array and microarray evaluation of endometrial receptivity in patients with high serum progesterone levels on the day of hCG administration. Reprod Biol Endocrinol 2011;9:29.

19. McKinnon B, Mueller M, Montgomery G. Progesterone Resistance in Endometriosis: an Acquired Property? Trends Endocrinol Metab 2018;29:535-48.

20. Haber E, Danenberg HD, Koroukhov N, et al.Peritoneal macrophage depletion by liposomal bisphosphonate

Cite this article as: D'Hooghe TM, Vanhie A, Flores VA, Taylor HS. Macrophage depletion: a potential immunomodulator treatment of endometriosis-associated pain? Ann Transl Med 2020;8(22):1534. doi: 10.21037/atm-2020-98 attenuates endometriosis in the rat model. Hum Reprod 2009;24:398-407.

21. Riccio LGC, Jeljeli M, Santulli P, et al. B lymphocytes inactivation by Ibrutinib limits endometriosis progression in mice. Hum Reprod 2019;34:1225-34.

22. Kotlyar A, Taylor HS, D'Hooghe TM. Use of immunomodulators to treat endometriosis. Best Pract Res Clin Obstet Gynaecol 2019;60:56-65.

23. Hogg C, Horne AW, Greaves E. Endometriosis-Associated Macrophages: Origin, Phenotype, and Function. Front Endocrinol (Lausanne) 2020;11:7.

24. D'Hooghe TM, Kyama CM, Chai D, et al. Nonhuman primate models for translational research in endometriosis. Reprod Sci 2009;16:152-61.

25. Vanhie A, Tomassetti C, Peeraer K, et al. Challenges in the development of novel therapeutic strategies for treatment of endometriosis. Expert Opin Ther Targets 2016;20:593-600. 